\title{
Characterization of a reproductive isolation gene, zygotic hybrid rescue, of Drosophila melanogaster by using minichromosomes
}

\author{
KYOICHI SAWAMURA* \& MASA-TOSHI YAMAMOTO $\dagger$ \\ Department of Biology, School of Education, Waseda University, Nishi-Waseda, Shinjuku, Tokyo, 169-50 and \\ †Department of Applied Biology, Kyoto Institute of Technology, Matsugasaki, Kyoto, 606, Japan
}

\begin{abstract}
Hybrids carrying the wild type allele of zygotic hybrid rescue (zhr) of Drosophila melanogaster and the maternal cytoplasm of its sibling species (D. simulans, $D$. mauritiana or $D$. sechellia) are embryonic lethal, irrespective of their sex. The $z h r$ gene has been localized in the $\mathrm{X}$ heterochromatin, slightly distal to the $\operatorname{In}(1) s c^{8}$ breakpoint, a location where highly repetitive satellite DNA exists. A free duplication minichromosome, $D p(1 ; f) 1162$, which is derived from the $\operatorname{In}(1) s c^{8}$ chromosome by a massive interstitial deletion has a significant, but not complete, effect on the hybrid lethality. In order to examine the organization of $z h r$ cytogenetically, we synthesized four deletions from $D p(1 ; f) 1162$ by $\gamma$-ray irradiation. Three of them, $D p(1 ; f) 1162 S 2, D p(1 ; f) 1162 S 3$, and $D p(1 ; f) 1162 S 5$, showed a hybrid lethal effect not significantly different from $D p(1 ; f) 1162$, but one, $D p(1 ; f) 1162 S 4$, showed a significantly weaker hybrid lethal effect. Interestingly, hybrids were completely lethal when two copies of $D p(1 ; f) 1162 S 4$ were present. That the lethal effect is proportional to the number of the minichromosomes supports the model that a class of repeated sequences around the $D p(1 ; f) 1162 S 4$ breakpoint may be involved in the $z h r$ gene activity. In the future, the minichromosome could be useful in the cloning and characterization of this reproductive isolation gene.
\end{abstract}

Keywords: Drosophila melanogaster, heterochromatin, hybrid inviability, minichromosome, reproductive isolation, zygotic hybrid rescue.

\section{Introduction}

It is important in evolutionary biology to show how reproductive isolation mechanisms develop between diverged populations (Mayr, 1963; Dobzhansky, 1970). So far, several isolating mechanisms have been elucidated genetically; in particular, genes causing postmating isolation have been mapped in several animal species (Wu \& Palopoli, 1994). For further analysis it is necessary to elucidate the molecular nature of these genes. One of the systems that has been analysed in detail is the lethal-hybrid rescue system in the Drosophila melanogaster species complex.

There are at least two types of lethality (embryonic and larval) in the hybrids between Drosophila

\footnotetext{
*Correspondence and present address: Department of Ecology and Evolution, University of Chicago, 1101 E. 57th Street, Chicago, IL 60637, U.S.A. E-mail: kysw@pondside.uchicago.edu
}

melanogaster and its sibling species (D. simulans, $D$. mauritiana and D. sechellia) (Sturtevant, 1920, 1929; Hadorn, 1961; David et al., 1974; Lachaise et al., 1986; Sawamura et al., 1993b). First, the hybrid males are lethal at the larval stage when crosses are made between $D$. melanogaster females and males of the sibling species. This lethality obeys Haldane's rule (Haldane, 1922) and has been explained by the conventional X chromosome/autosome (X/A) imbalance hypothesis: i. e. hybrids carrying a haploid set of autosomes from the sibling species without a conspecific X chromosome are lethal (Yamamoto, 1992; Orr, 1993; Sawamura et al., 1993a,c; Wu \& Davis, 1993). They are rescued by either Hybrid male rescue (Hmr) of D. melanogaster (Hutter \& Ashburner, 1987; Hutter et al., 1990) or Lethal hybrid rescue (Lhr) of D. simulans (Watanabe, 1979; Takamura \& Watanabe, 1980). These genes are thought to compensate for the $\mathrm{X} / \mathrm{A}$ imbalance in the hybrids. Secondly, embryonic lethality is observed in the 
hybrid females from the crosses between females of the sibling species and males of $D$. melanogaster; this contradicts Haldane's rule. This lethality is caused by the existence of a D. melanogaster $\mathrm{X}$ chromosome together with the maternal cytoplasm of the sibling species (Sawamura et al., 1993a,c). The hybrids are rescued by zygotic hybrid rescue (zhr) of D. melanogaster (Sawamura et al., 1993c) or maternal hybrid rescue $(\mathrm{mhr})$ of $D$. simulans (Sawamura et al., 1993a). Wild-type alleles of these loci are thought to contribute to the maternal gene/ $\mathrm{X}$ chromosome incompatibility (Sawamura, 1996).

In this study we use chromosomal aberrations (duplications and deficiencies) of D. melanogaster to map these genes cytogenetically. Previously, chromosomal aberrations have helped to map one of the larval lethal hybrid rescue genes, $\mathrm{Hmr}$ (Hutter et al., 1990; Hutter \& Karch, 1994). In our own studies, we have mapped a gene involved in the hybrid embryonic lethality, $z h r$, to a specific region of the $\mathrm{X}$ heterochromatin. We used minichromosomes which carry various amounts of $\mathrm{X}$ heterochromatin (Sawamura \& Yamamoto, 1993). Although we concluded that the location of the $z h r$ gene is between the breakpoints of $D p(1 ; f) 1162$ and $D p(1 ; f) 1205$, the former, $D p(1 ; f) 1162$, itself had a partial effect on the lethality. A fraction of viables was consistently recovered in the hybrids carrying this minichromosome (Sawamura \& Yamamoto, 1993; Sawamura et al., 1995). This may mean that $z h r$ gene activity is quantitative.

In order to examine this quantitative level hypothesis, we induced four distal heterochromatic deficiencies in $D p(1 ; f) 1162$, and investigated the strength of their hybrid lethal effect. Three of them, $D p(1 ; f) 1162 S 2, D p(1 ; f) 1162 S 3$ and $D p(1 ; f) 1162 S 5$, showed the effect not significantly different from the original $D p(1 ; f) 1162$, whereas the other deleted minichromosome, $D p(1 ; f) 1162 S 4$, showed significantly weakened hybrid lethal effect. Interestingly, two doses of this minichromosome made hybrids completely lethal. This suggests that a class of repeated sequence(s) may be involved in the $z h r$ gene and that the sequence(s) should be distributed around the $D p(1 ; f) 1162 S 4$ breakpoint.

\section{Materials and methods}

\section{Cytology}

Chromosome preparations were made and minichromosome size relative to the fourth chromosome was measured according to the method described previously (Park \& Yamamoto, 1993).

\section{Interspecific crosses}

Females of a Drosophila sechellia strain, $y^{\text {sim }}$ ( $y$ mutation introgressed from $D$. simulans), were used for interspecific crosses. All crosses were performed at $23^{\circ} \mathrm{C}$.

\section{The viability of hybrids carrying minichromosomes}

The viability of hybrid males carrying different numbers of minichromosomes was estimated as (No. observed)/(No. expected). The expected number was calculated from the distribution of minichromosome number in larvae which were brothers of the paternal strains used for the tests and was based on the three assumptions: (i) the probability that a father carrying $n$ copies of a minichromosome generates hybrids inheriting $r$ copies of it is ${ }_{n} \mathrm{C}_{\mathrm{r}} / 2^{n}$; (ii) all classes of fathers, regardless of their minichromosome number, contribute to the production of hybrids proportionally; and (iii) the expected number of fully viable hybrids which do not carry the minichromosome is adjusted to be equal to the actual number of hybrids that survived.

\section{Results}

Screening for deletions from the minichromosome $\mathrm{Dp}(1 ; \mathrm{f}) 1162$

$D p(1 ; f) 1162$ of Drosophila melanogaster is a free duplication minichromosome derived from the In (1) $s c^{8}$ chromosome by an interstitial deletion (Fig. 1; see Lindsley \& Zimm, 1992). It carries a tiny tip of the $\mathrm{X}$ euchromatin ranging from the terminus to the $a c$ locus $[l(1) 1 A a, l(1) 1 A c$, cin, ewg, $y$ and $a c]$ and a part of the heterochromatin which originates from two portions: (i) a segment slightly distal to the $\ln (1) s c^{8}$ breakpoint which includes the $z h r$ gene, and (ii) the centromeric region (Fig. 1). To obtain a series of minichromosomes deleted for the euchromatin-heterochromatin junction region, we screened for minichromosomes carrying a deletion for $y^{+}$but retaining vital genes distal to it [which complement $D f(1) 259]$. The loss of $y^{+}$is a good marker for a possible deletion of the distal heterochromatic region near which the $z h r$ gene is located.

Males of wild type body colour whose genotype was $X Y L . Y S, D f(1) 259, y w / D p(1 ; f) 1162, y^{+} / 0$ were exposed to $\gamma$-rays $(3500 \mathrm{rad})$, and were crossed to $C(1) R M, y \vee f / 0$ or $F M 7 / X Y L . Y S, D f(1) 259, y w$ females (Fig. 2). [Note that $D p(1 ; f) 1162$ segregates randomly from the sex chromosomes (H-S. Park \& M-T. Yamamoto, unpublished).] Eight yellow white 


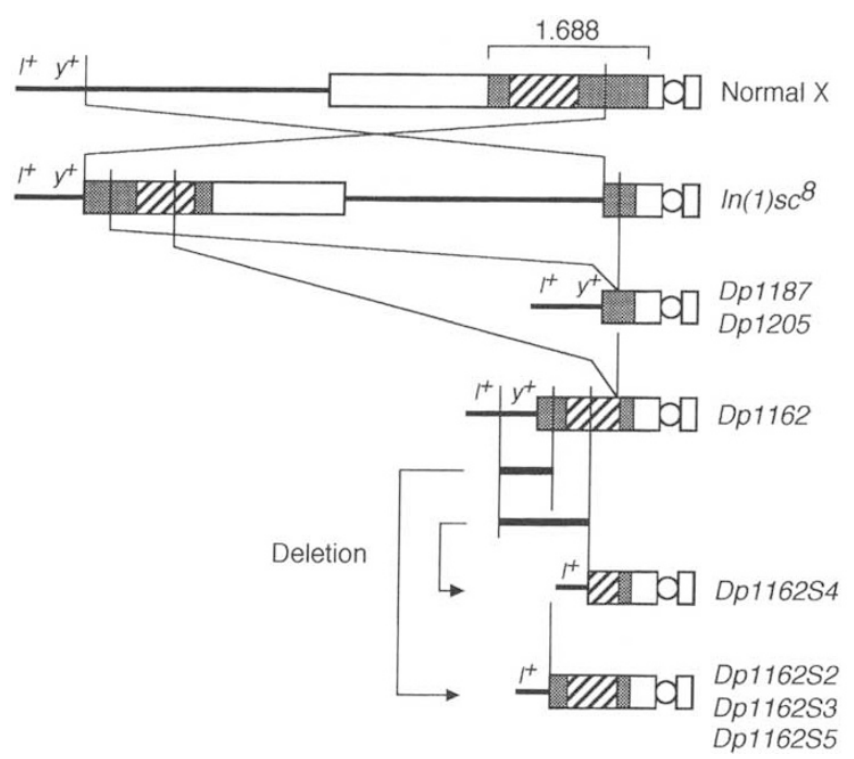

Fig. 1 Diagrammatic representation of minichromosome synthesis in Drosophila. Euchromatin, heterochromatin, and the centromere are shown by the solid line, boxes, and the circle, respectively. The region rich in $1.688 \mathrm{~g}$ $\mathrm{cm}^{-3}$ satellite DNA (1.688) and the presumed location of the $z h r$ gene (hatched box) are indicated. Genes located on the tip of the euchromatin - essential genes $\left(l^{+}\right)$ [which represent $l(1) 1 A a, l(1) 1 A c, c i n$, and $e w g$ ] and a marker gene $\left(y^{+}\right)$- are also shown. $D p$ represents $D p(1 ; f)$.

males were obtained, and were individually crossed to $C(1) R M, y v f / 0$ females to establish new stocks. Two were sterile and another two were each accompanied by a cytologically visible $y$ translocation. The remaining four had minichromosomes which are thought to be deficient for $y^{+}$. These four new minichromosomes were named $D p(1 ; f) 1162 S 2$, $D p(1 ; f) 1162 S 3, D p(1 ; f) 1162 S 4$ and $D p(1 ; f) 1162 S 5$.

\section{Characterization of newly synthesized minichromosomes}

None of these new minichromosomes complemented either $y$ or $a c$ mutations, indicating the deletion of the most proximal euchromatic portion of $D p(1 ; f) 1162$. Their distal breakpoints are thought to lie in a small region between $y$ and ewg (the most proximal vital gene included in $D f(1) 259$, unpublished). In fact, it was shown that all of these new minichromosomes complement the lethality of $e w g$ and the cin maternal effect.

Furthermore, the most distal portion of the heterochromatin may have also been deleted in these new minichromosomes, because the length of these minichromosomes was cytologically shorter

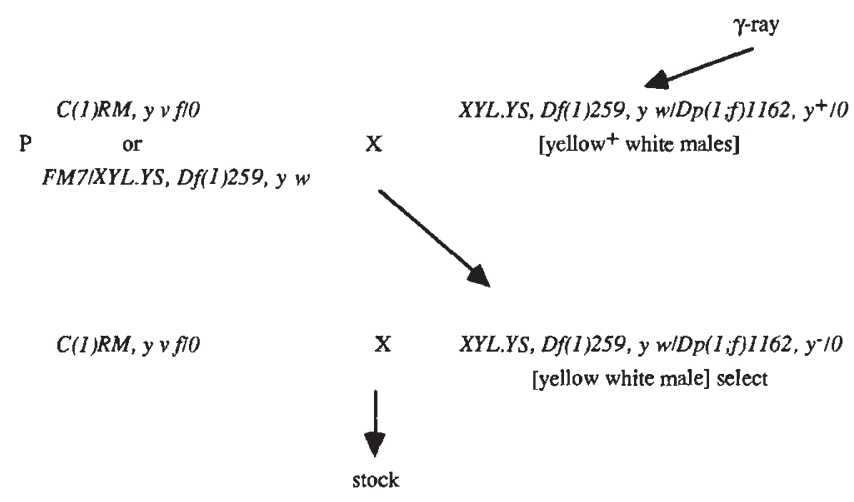

Fig. 2 Mating scheme for screening deletions from Dp $(1 ; f) 1162$.

Table 1 Length of minichromosomes relative to the fourth chromosome

\begin{tabular}{lc}
\hline Minichromosome & Length $\pm \mathrm{SE}$ \\
\hline$D p(1 ; f) 1162 \dagger$ & $0.45 \pm 0.03$ \\
$D p(1 ; f) 1162 S 2$ & $0.39 \pm 0.02$ \\
$D p(1 ; f) 1162 S 3$ & $0.36 \pm 0.03$ \\
$D p(1 ; f) 1162 S 4$ & $0.36 \pm 0.03$ \\
$D p(1 ; f) 1162 S 5$ & $0.38 \pm 0.03$ \\
\hline
\end{tabular}

†Data after Sawamura \& Yamamoto (1993).

than the original $D p(1 ; f) 1162$ (Table 1). Although the exact locations of the proximal breakpoints are unknown, it has been shown that the heterochromatic breakpoints are within the region rich in $1.688 \mathrm{~g} / \mathrm{cm}^{-3}$ satellite DNA (Fig. 1): the satellite DNA exists not only in the heterochromatin of $D p(1 ; f) 1162$ (Sawamura et al., 1995) but also in the heterochromatin of all these new minichromosomes (data not shown).

\section{The zhr gene activity of new minichromosomes}

We examined the newly synthesized minichromosomes whether the $z h r$ gene is retained or not, by investigating the viability of hybrids carrying these minichromosomes. Our observations indicated that all of them contained the gene activity (i. e. causing embryonic lethality) to some extent: (i) no dead embryos were seen in the cross between Drosophila sechellia $y^{\text {sim }}$ females and YSX.YL, In (1)EN, y B/0 males, which indicates that this attached $X Y$ chromosome is deficient for the $z h r$ gene (negative control); (ii) a class of dead embryos showing the typical phenotype (Sawamura et al., 1993b) was produced when YSX.YL, $\operatorname{In}(1) E N, y$ 
$B / D p(1 ; f) 1162, y^{+} / 0$ were used, which indicates that the original minichromosome carried the gene (positive control); (iii) when a mixture of either YSX.YL, In (1)EN, $y \quad B / D p(1 ; f) 1162 S, y^{-} / 0$ or $Y S X . Y L$, In (1) $E N, y B / 0$ was used (we cannot follow the existence of the minichromosome phenotypically), all newly synthesized minichromosomes caused embryonic lethality.

In order to measure the strength of the $z h r$ gene activity in the new minichromosomes, we crossed D. sechellia $y^{\text {sim }}$ females to XYL.YS, Df (1)259, y $w / D p(1 ; f) 1162 S / 0$ males, and analysed the viable hybrid male larvae cytologically. Because the lethality of $D f(1) 259$ is complemented by the minichromosome, the paternal males should carry at least one minichromosome [note that they may have accumulated more than one by random segregation (Yamamoto \& Miklos, 1977)]. If one or more mini- chromosomes are detected in viable male hybrids, it is assumed that the minichromosome lacks the $z h r$ gene or has less gene activity. It would be ideal to make the interspecific crosses using a single male whose minichromosome number had been checked by an intraspecific control cross. But we were compelled to make interspecific crosses en masse, to overcome the low success rate of interspecific crosses. To estimate the expected numbers of hybrids carrying various minichromosome numbers, we determined the frequency in male larvae from the same population which we used for the test cross (Table 2) (see Materials and methods for the calculation).

The interspecific cross data (see the relative viability of hybrids bearing the minichromosomes shown in Table 3 ) show that the hybrid lethal effects of $D p(1 ; f) 1162 S 3$ and $D p(1 ; f) 1162 S 5$ are lower

Table 2 Frequencies of males bearing various numbers of minichromosomes in stocks

\begin{tabular}{llcccr}
\hline & \multicolumn{3}{c}{ No. of duplication } \\
\cline { 2 - 5 } Minimichrosome & $D p$ & $D p / D p$ & $D p / D p / D p$ & $D p / D p / D p / D p$ & Mean \\
\hline$D p(1 ; f) 1162 \dagger$ & $6 \ddagger$ & $18 \ddagger$ & 5 & 0 & 1.97 \\
$D p(1 ; f) 1162 S 2$ & 0 & 10 & $6 \ddagger$ & 0 & 2.38 \\
$D p(1 ; f) 1162 S 3$ & 2 & 8 & 6 & 0 & 2.44 \\
$D p(1 ; f) 1162 S 4$ & 1 & 17 & 5 & 1 & 2.17 \\
$D p(1 ; f) 1162 S 5$ & 1 & 10 & 11 & & 2.52 \\
\hline
\end{tabular}

The genotype of stocks is $C(1) R M, y v f / X Y L . Y S, D f(1) 259, y w / D p(1 ; f) / 0$.

†Data after Sawamura et al. (1995).

¥One was triplo-4.

Table 3 Frequencies of hybrid males bearing various numbers of minichromosomes from the cross between $D$. sechellia $y^{\mathrm{sim}}$ females and $X Y L . Y S, D f(1) 259, y w / D p(1 ; f) / 0$ males and relative viability of hybrids with the minichromosomes

\begin{tabular}{|c|c|c|c|c|c|}
\hline \multirow[b]{2}{*}{ Minimichrosome } & & \multicolumn{3}{|c|}{ No. of duplication } & \multirow[b]{2}{*}{ Mean } \\
\hline & & 0 & $D p$ & $D p / D p$ & \\
\hline \multirow[t]{2}{*}{$D p(1 ; f) 1162 \dagger$} & Obs. & 46 & $5(0.06)$ & $0(0)$ & \multirow[t]{2}{*}{0.10} \\
\hline & Exp. & 46 & 78.6 & 36.1 & \\
\hline \multirow[t]{2}{*}{$D p(1 ; f) 1162 S 2$} & Obs. & $28 \ddagger$ & $0(0)$ & $1(0.02)$ & \multirow[t]{2}{*}{0.07} \\
\hline & Exp. & 28 & 62.5 & 40.9 & \\
\hline \multirow[t]{2}{*}{$D p(1 ; f) 1162 S 3$} & Obs. & 27 & $8(0.15)$ & $1(0.03)$ & \multirow[t]{2}{*}{0.28} \\
\hline & Exp. & 27 & 54.0 & 34.8 & \\
\hline \multirow[t]{2}{*}{$D p(1 ; f) 1162 S 4$} & Obs. & 17 & $16(0.47)$ & $0(0)$ & \multirow[t]{2}{*}{0.48} \\
\hline & Exp. & 17 & 34.4 & 19.4 & \\
\hline \multirow[t]{2}{*}{$D p(1 ; f) 1162 S 5$} & Obs. & 23 & $8(0.16)$ & $0(0)$ & \multirow[t]{2}{*}{0.26} \\
\hline & Exp. & 23 & 51.2 & 36.3 & \\
\hline
\end{tabular}

Relative viability is shown in parentheses.

Expected values were calculated based on Table 2 (see Materials and methods).

†Data after Sawamura et al. (1995).

†One was haplo-4. 
than those of $D p(1 ; f) 1162 S 2$ and the original $D p(1 ; f) 1162$ but the difference is not significant. $D p(1 ; f) 1162 S 4$ showed a significantly weaker hybrid lethal effect (Table 3 ). The viability of hybrid males carrying one copy of $D p(1 ; f) 1162 S 4$ was 0.47 . But we found no hybrid males carrying two or more copies of $D p(1 ; f) 1162 S 4$; i.e. this minichromosome had a dose-dependent hybrid lethal effect. A class of repeated sequences around the $D p(1 ; f) 1162 S 4$ breakpoint may be involved in the hybrid lethality, and the dose-dependent effect suggests that a quantitative level of the sequences is necessary to cause complete hybrid lethality.

\section{Discussion}

\section{Genetic nature of the zhr gene}

The crosses between Drosophila melanogaster males and females of its sibling species ( $D$. simulans, $D$. mauritiana or $D$. sechellia) produce hybrids which are lethal in embryos when they carry the wild-type allele of an X-linked heterochromatic gene, zygotic hybrid rescue (zhr) of D. melanogaster (Sawamura et al., 1993c). A minichromosome, $D p(1 ; f) 1162$, which was derived from the $\operatorname{In}(1) s c^{8}$ chromosome by an interstitial deletion, carries partial $z h r$ gene activity (Sawamura \& Yamamoto, 1993; Sawamura et al., 1995). For further genetic characterization of the gene, flies carrying $D p(1 ; f) 1162$ were exposed to $\gamma$-rays to produce new minichromosomes each of which had a deletion in the euchromatin-heterochromatin junction near which the $z h r$ gene is located.

Smaller minichromosomes, $D p(1 ; f) 1162 S 3$ and $D p(1 ; f) 1162 S 5$, showed lower $z h r$ gene activity than $D p(1 ; f) 1162 S 2$ and the original $D p(1 ; f) 1162$, but the difference was not significant. One of the smallest minichromosomes, $D p(1 ; f) 1162 S 4$, showed a significantly weaker hybrid lethal effect. There remains a possibility that this weakened effect may be caused by position effect variegation, as the deficiency brought the heterochromatic $z h r$ gene closer to euchromatin. However, our preliminary data did not support this idea: the effect was not modified by a $\mathrm{Y}$ chromosome (data not shown) which is a general modifier of position effect variegation (Spofford, 1976). Further, hybrids carrying two or more copies of $D p(1 ; f) 1162 S 4$ were completely lethal, showing a dose-dependent effect. Based on several lines of evidence above, namely (i) partial effect of $D p(1 ; f) 1162$; (ii) gradation from $D p(1 ; f) 1162$; (iii) correlation with physical size (though not significant); and (iv) dose effect of $D p(1 ; f) 1162 S 4$, we assume that a class of repetitive sequences may be involved in the $z h r$ gene and that $D p(1 ; f) 1162 S 4$ may carry lesser amounts of the sequences than $D p(1 ; f) 1162$.

\section{Perspective for finer resolution mapping of the zhr gene}

The $z h r$ gene has been mapped in a heterochromatic region proximal to the $\operatorname{In}(1) s c^{L 8}$ breakpoint but distal to the $\operatorname{In}(1) s c^{8}$ breakpoint (Sawamura \& Yamamoto, 1993). Because $D p(1 ; f) 1162$ is a minichromosome derived from $\operatorname{In}(1) s c^{8}$ by a large interstitial deletion, it does not contain a heterochromatic portion distal to the $z h r$ gene in the normal X chromosome order (Fig. 1). The $D p(1 ; f) 1162$ chromosome is supposed to be partially deleted for the repetitive sequences involved in the $z h r$ gene, as has been discussed. This means that $D p(1 ; f) 1162$ lacks the sequences proximally in the (inverted) minichromosome order. In the present screening we tried to get smaller minichromosomes which are deleted for more of these sequences distally. None of four derived minichromosomes completely excluded the hybrid lethal effect, although we initially expected to recover such minichromosomes. This indicates that the sequences are not restricted to the most distal heterochromatic region of $D p(1 ; f) 1162$ (i. e. the juxtaposition to the heterochromatic breakpoint of $\left.\operatorname{In}(1) s c^{8}\right)$. Alternatively, they would be at a position slightly interior (distal to the $\operatorname{In}(1) s c^{8}$ breakpoint in the normal $\mathrm{X}$ order). This is consistent with the fact that $l(1) J 1^{+} Y$, which was derived by a similar deletion from $y^{+} Y$, retains the complete hybrid lethal effect (unpublished).

In conjunction with our previous paper (Sawamura et al., 1995), it is supposed that unknown repetitive sequences scattered in a specific site of a $1.688 \mathrm{~g} \mathrm{~cm}^{-3}$ satellite DNA rich region are involved in the $z h r$ gene. It is informative that $D p(1 ; f) 1162 S 4$ may partially carry the sequences on the most distal portion of its heterochromatin. Recently, it was shown that complex sequence islands exist in the 1.688 satellite DNA rich region (Le et al., 1995). According to the construction history of minichromosomes (Fig. 1), $D p(1 ; f) 1162$ carries a larger 1.688 satellite DNA rich region than $D p(1 ; f) 1187$ between two complex sequence islands, Tahiti and Moorea, so the $z h r$ gene is supposed to be localized between these two islands. Because the distal island Tahiti consists of a retroposon Doc (Le et al., 1995), it is intriguing to know whether new deficiency minichromosomes derived from $D p(1 ; f) 1162$ retain the 
sequence as detected in the original minichromosome by in situ hybridization (Carmena \& González, 1995). These molecular and cytological studies will provide useful information for cloning the $z h r$ gene.

\section{Molecular model of the hybrid embryonic lethality}

Hybrids produced by the sibling species mothers are embryonic lethal when they inherit the wild-type allele of $z h r$ from $D$. melanogaster, because of the incompatibility with a maternal effect gene(s). The lethal hybrids can be rescued maternally by the $m h r$ mutation (Sawamura et al., 1993a). Although there remains a possibility that the $z h r$ gene codes a unique protein, it is an attractive hypothesis that this region, which may consist of a class of repeated sequences, is a binding site of the $m h r$ gene products. There is also a possibility that sequences homologous to the $z h r$ region exist in other regions, as promoters of some essential genes. The following model is possible: $m h r$ of $D$. melanogaster produces DNA-binding proteins (transcription factors) enough to titrate the $z h r$ region, but the wild-type allele of the sibling species $m h r$ produces less of it because of the absence of the $z h r$ region in these species. In this model, the $z h r$ region of $D$. melanogaster traps all products of the wild-type allele of the sibling species $m h r$, and the hybrids become lethal.

\section{Acknowledgements}

We are grateful to H. Miura and Y. Saito for $\gamma$-ray irradiation, to A. T. C. Carpenter and K. White for providing us fly stocks, to $\mathrm{Y}$. $\mathrm{H}$. Inoue and $\mathrm{H}$-S. Park for their technical advice, to A. Fujita and R. Yokoyama for their technical assistance, to T. Taira for his kind support, to M. F. Palopoli for his advice on statistics, and to M. Ashburner, A. W. Davis, and C.-I Wu for their comments on the various versions of the manuscript. This research was supported by Waseda University Grant for Special Research Projects to K. S.

\section{References}

CARMENA, M. AND GONZÁlez, C. 1995. Transposable elements map in a conserved pattern of distribution extending from beta-heterochromatin to centromeres in Drosophila melanogaster. Chromosoma, 103, 676-684.

DAVID, J. R., LEMEUNIER, F., TSACAS, L. AND BOCQUET, C. 1974. Hybridation d'une nouvelle espèce, Drosophila mauritiana avec $D$. melanogaster et $D$. simulans. Ann. Genet., 17, 235-241.
DOBZHANSKY, TH. 1970. Genetics of the Evolutionary Process. Columbia University Press, New York.

HAdOrn, E. 1961. Zur Autonomie und Phasenspecifität der Letalität von Bastarden zwischen Drosophila melanogaster und Drosophila simulans. Rev. Suisse Zool., 68, 197-207.

HALDANE, J. B. S. 1922. Sex ratio and unisexual sterility in hybrid animals. J. Genet., 12, 101-109.

HUTTER, P. AND ASHBURNER, M. 1987. Genetic rescue of inviable hybrids between Drosophila melanogaster and its sibling species. Nature, 327, 331-333.

HUTTER, P. AND KARCH, F. 1994. Molecular analysis of a candidate gene for the reproductive isolation between sibling species of Drosophila. Experientia, 50, 749-762.

HUTTER, P., ROOTE, J. AND ASHBURNER, M. 1990. A genetic basis for the inviability of hybrids between sibling species of Drosophila. Genetics, 124, 909-920.

LACHAISE, D., DAVID, J. R., LEMEUNIER, F., TSACAS, L. AND ASHBURNER, M. 1986. The reproductive relationships of Drosophila sechellia with D. mauritiana, D. simulans, and $D$. melanogaster from the Afrotropical region. Evolution, 40, 262-271.

LE, M.-H., DURICKA, D. AND KARPEN, G. H. 1995. Islands of complex DNA are widespread in Drosophila centric heterochromatin. Genetics, 141, 283-303.

LINDSLEY, D. L. AND ZIMM, G. G. 1992. The Genome of Drosophila melanogaster. Academic Press, San Diego.

MAYR, E. 1963. Animal Species and Evolution. Belknap, Cambridge, MA.

ORR, H. A. 1993. Haldane's rule has multiple genetic causes. Nature, 361, 532-533.

PARK, H-S. AND yAmamoto, M-T. 1993. Synthesis of free $X$ duplications carrying a specific region of the centromeric heterochromatin in Drosophila melanogaster. Jap. J. Genet., 68, 83-95.

SAWAMURA, K. 1996. Maternal effect as a cause of exceptions for Haldane's rule. Genetics, 143, 609-611.

SAWAMURA, K. AND YAMAMOTO, M-T. 1993. Cytogenetical localization of Zygotic hybrid rescue $(Z h r)$, a Drosophila melanogaster gene that rescues interspecific hybrids from embryonic lethality. Mol. Gen. Genet., 239, $441-449$.

SAWAMURA, K., TAIRA, T. AND WATANABE, T. K. 1993a. Hybrid lethal systems in the Drosophila melanogaster species complex. I. The maternal hybrid rescue ( $\mathrm{mhr}$ ) gene of Drosophila simulans. Genetics, 133, 299-305.

SAWAMURA, K., WATANABE, T. K. AND YAMAMOTO, M-T. 1993b. Hybrid lethal systems in the Drosophila melanogaster species complex. Genetica, 88, 175-185.

SAWAMURA, K., YAMAMOTO, M-T. AND WATANABE, T. K. 1993c. Hybrid lethal systems in the Drosophila melanogaster species complex. II. The Zygotic hybrid rescue (Zhr) gene of D. melanogaster. Genetics, 133, 307-313.

SAWAMURA, K., FUJITA, A., YOKOYAMA, R., TAIRA, T, INOUE, Y. H., PARK, H-S. AND YAMAMOTO, M-T. 1995. Molecular and genetic dissection of a reproductive isolation gene, zygotic hybrid rescue, of Drosophila melanogaster. Jap. J. Genet., 70, 223-232. 
SPOFFORD, J. B. 1976. Position-effect variegation in Drosophila. In: Ashburner, M. and Novitski, E. (eds) The Genetics and Biology of Drosophila, vol. 1c, pp. 955-1018. Academic Press, London.

Sturtevant, A. H. 1920. Genetic studies on Drosophila simulans. I. Introduction. Hybrids with Drosophila melanogaster. Genetics, 5, 488-500.

sturtevant, A. H. 1929. The genetics of Drosophila simulans. Carnegie Inst. Wash. Publ., 399, 1-62.

TAKAMURA, T. AND WATANABE, T. K. 1980. Further studies on the Lethal hybrid rescue ( $L \mathrm{hr}$ ) gene of Drosophila simulans. Jap. J. Genet., 55, 405-408.

WATANABE, T. K. 1979. A gene that rescues the lethal hybrids between Drosophila melanogaster and D. simulans. Jap. J. Genet., 54, 325-331.
WU, C.-1 AND DAvis, A. w. 1993. Evolution of postmating reproductive isolation: the composite nature of Haldane's rule and genetic bases. Am. Nat., 142, 187-212.

WU, C.-1 AND PALOPOLI, M. F. 1994. Genetics of postmating reproductive isolation in animals. Ann. Rev. Genet., 28, 283-308.

YАMАMOTо, M-т. 1992. Inviability of hybrids between $D$. melanogaster and $D$. simulans results from the absence of simulans $X$ not the presence of simulans $Y$ chromosome. Genetica, 87, 151-158.

YAMAMOTO, M. AND M1KLOS, G. L. G. 1977. Genetic dissection of heterochromatin in Drosophila: the role of basal $\mathrm{X}$ heterochromatin in meiotic sex chromosome behaviour. Chromosoma, 60, 283-296. 